\title{
Oddball onset timing: Little evidence of early gating of oddball stimuli from tapping, reacting, and producing
}

\author{
Jordan Wehrman $^{1}$ (D) Paul Sowman ${ }^{1}$ \\ Accepted: 21 January 2021 / Published online: 15 March 2021 \\ (C) The Psychonomic Society, Inc. 2021
}

\begin{abstract}
Oddballs, rare or novel stimuli, appear to last longer than non-oddballs. This illusion is often attributed to the perceived time that an oddball occupies being longer than that of a non-oddball. However, it is also possible that oddball stimuli are perceived to onset earlier than non-oddballs; they are "gated" earlier in time and thus the perceived duration of those stimuli are longer. In the current article, we directly investigate this proposal by asking participants to react to, produce durations initiated with, and tap along to either oddball or standard stimuli. Tapping provided some support for earlier perceived onset of an oddball in the visual modality. However, both reaction time and duration production experiments provided evidence against an oddball being gated earlier than a standard stimulus. Contrarily, these experiments showed an oddball resulted in longer reaction times and productions, respectively. Taken together, these three experiments indicate it is unlikely that the expansion of time attributed to oddball presentation is purely due to the earlier gating of oddball stimuli. In fact, the first two experiments provide some evidence that the effect of an oddball must compensate for the later gating of these stimuli.
\end{abstract}

Keywords Reaction time methods $\cdot$ Temporal processing $\cdot$ Visual perception

\section{Introduction}

Subjectively, time is relatively malleable; perceived duration can expand or contract depending on several factors. For example, a larger magnitude stimulus tends to be perceived to last longer than a smaller magnitude stimulus (Alards-Tomalin, LeboeMcGowan, Shaw, \& Leboe-McGowan, 2014; Cai \& Connell, 2016; Rammsayer \& Verner, 2014; Wehrman, Kaplan, \& Sowman, 2020a; Xuan, Chen, He, \& Zhang, 2009), and prior judgments affect the reported durations of a current stimulus (Wehrman, Wearden, \& Sowman, 2018a, b; Wehrman et al., 2020a; Wehrman, Wearden, \& Sowman, 2020b; Wehrman \& Sowman, 2019; Wiener, Thompson, \& Coslett, 2014).

Recently, another manipulation of perceived duration has been gaining attention, the so-called "oddball duration" illusion. In this illusion, participants are asked to judge the duration of either standard/repeated stimuli or rare/novel (i.e., oddball) stimuli. Generally, it has been found that an oddball stimulus is perceived to last longer than a non-oddball stimulus (Alexander et al., 1995;

Jordan Wehrman

Jordan.wehrman@mq.edu.au

1 Cognitive Sciences, Macquarie University, Sydney, NSW, Australia
Alexander et al., 2016; Birngruber, Schröter, \& Ulrich, 2014; Fromboluti, Jones, \& McAuley, 2013; Schindel, Rowlands, \& Arnold, 2011; Tse, 2010; Tse, Intriligator, Rivest, \& Cavanagh, 2004; Wehrman, 2020a; Wehrman et al., 2018a, b).

Two mechanisms have been proposed to account for the generation of this temporal expansion. The most commonly cited is that an oddball occupies more subjective time relative to the comparison stimulus. This has variously been attributed to an oddball expanding time (see Matthews \& Gheorghiu, 2016; Penney, 2003) or a repeated stimulus compressing it (see Eagleman \& Pariyadath, 2009; Pariyadath \& Eagleman, 2007). However, early gating of an oddball stimulus, i.e., an earlier perceived onset, has also been proposed to expand the relative duration of an oddball (Lin \& Shimojo, 2017; McAuley \& Fromboluti, 2014; Wehrman et al., 2018a, b). Essentially, if a non-oddball takes $30 \mathrm{~ms}$ to register while an oddball takes 10 $\mathrm{ms}$, the oddball will be perceived to last $20 \mathrm{~ms}$ longer than the non-oddball stimulus (note that timing seems to occur from perceived onset; see Wehrman, 2020b).

Most investigations of early gating have assessed the claim relatively indirectly. For example, Wehrman et al. (2018a, b) used variable foreperiods to show a role of expectation in the perceived duration of an oddball stimulus, attributing the effects of attention to an earlier perceived onset of the oddball stimulus. However, it is possible that simply expecting a 
stimulus to occur improves the processing of that stimulus, resulting in biased processing of the expected stimulus and thus an expanded perceived duration (see Matthews \& Gheorghiu, 2016). Instead of using indirect measures of oddball onset, in the current article we directly assess when an oddball is perceived to onset. Specifically, we investigate whether online earlier gating occurs when an oddball stimulus is presented. By this we mean that an oddball is genuinely perceived sooner than a non-oddball stimulus, rather than an oddball stimulus being remembered to have begun earlier in time. Before introducing the methods used in the current article, we will briefly discuss one attempt to examine early gating of oddball stimuli using simultaneity judgments.

\section{Oddball synchrony}

In a recent article, Wehrman (2020a) attempted to find evidence for early perception of oddball onset by using a simultaneity judgment task. In this task, participants were required to judge whether a stimulus (either a light or a sound) occurred simultaneously with another stimulus in the opposite modality (either a sound or a light, respectively). In the target modality, the stimulus was either repeated or different (Experiment 1) or common or rare (Experiment 2). In both experiments, using Bayesian statistics, Wehrman (2020a) found moderate evidence against an oddball having an earlier perceived onset compared to a non-oddball stimulus.

However, simultaneity judgments may not be the ideal tool for uncovering differences in perceived onset for several reasons. Firstly, in simultaneity judgment tasks, the stimuli are presented at various asynchronies from one another, then on each trial participants judge whether the asynchrony was zero (i.e., simultaneous) or not. However, these asynchronies present a discontinuous measure of perceived onset. Compounding on this temporal "blur," the perceived onset of any given oddball is likely to vary from trial to trial. This means that the perceived onset of an oddball, as measured by any given asynchrony, may be somewhat unreliable. Indeed, given the width of the perceived onset synchrony curve, there appears to be evidence for such a blurring. Additionally compounding these issues, simultaneity judgments are made after the actual stimuli have been presented, and thus may be subject to post-stimulus rationalization. Thus, it may be that coincidence detection is not adequate for the detection of onset differences between oddball and standard stimuli.

\section{Current approach}

In the current article, three different approaches are taken to investigate perceived oddball onset. Each of these present oddballs without requiring the direct judgment of duration or onset. We will introduce the three tasks in turn. The general task format is depicted in Fig. 1.

\section{Oddball reactions}

In the first experiment, participants performed a simple reaction time (RT) task. In this task, participants were required to reactively respond to a stimulus in either the auditory or the visual modality. Because performing a different response to either an oddball or a standard stimulus would alter RTs due to the relative rarity of oddballs, a single response was required whichever stimulus was presented.

If an oddball is perceived to appear earlier than a standard stimulus, we predict that RTs will be shorter to an oddball stimulus compared to a standard stimulus. This is because, if both stimuli objectively appear at the same time, and there are no other factors that speed up responses for one stimulus in comparison to another, then the stimulus that is perceived earlier will be responded to earlier. However, the previous trial tends to also affect RTs. For example, if the imperative appears earlier in the current trial compared to the last trial, RTs tend to be much longer (Grondin \& Rammsayer, 2003; Los, 2010; Steinborn, Rolke, Bratzke, \& Ulrich, 2008; Thomaschke, Wagener, Kiesel, \& Hoffmann, 2011; Vallesi, Lozano, \& Correa, 2013). Therefore, we analyzed RTs across three conditions, when the current and prior stimulus were both standard (S-S), when the prior stimulus was an oddball and the current stimulus was a standard (O-S), and when the prior trial was a standard stimulus and the current stimulus was an oddball (S-O). We predict that when the current trial contains an oddball stimulus and the prior trial contains a standard stimulus, RTs will be faster than in the other two conditions. Further, perhaps the oddball will serve to grab

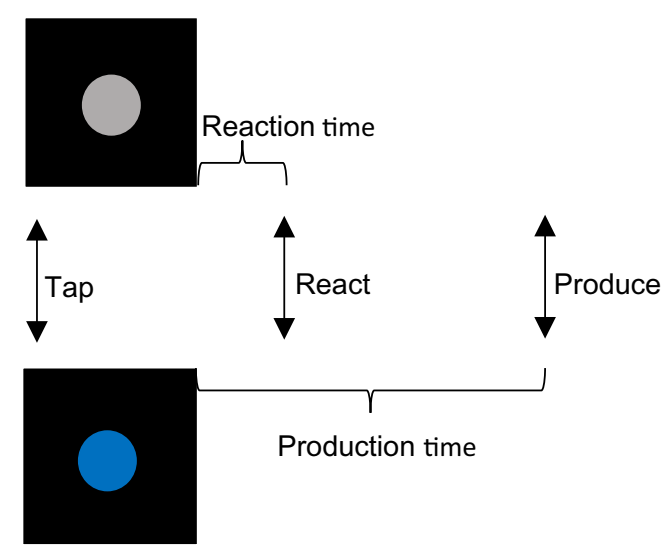

Fig. 1 General outline of the three tasks used here. One of these two stimuli was the standard and one was the oddball. In Experiment 1, participants reacted as quickly as possible to the stimuli. In Experiment 2, participants waited a set time, a temporal production, then terminated the production with a button press. In Experiment 3, participants tapped in time with these stimuli 
the participants' attention, which may feed over to the subsequent trial, resulting in O-S RTs being the second fastest.

\section{Oddball productions}

In the second task, participants were asked to produce various durations ( $500 \mathrm{~ms}, 750 \mathrm{~ms}, 1,000 \mathrm{~ms}, 1,250 \mathrm{~ms}$ ) by terminating an automatically initiated interval. The majority of the stimuli initiating a production were standard; however, occasionally the stimuli were deviant oddballs. In this case, if an oddball is perceived to begin earlier, then productions should be shorter than when the production is initiated with a standard stimulus. This is because, relative to the objective starting point (i.e., when either the oddball or the standard was presented) the oddball will be perceived earlier. If the perceived onset of the interval is earlier, then the entire production should be shifted to an earlier point in time, resulting in a shorter duration being produced.

\section{Oddball tapping}

In this final task, participants tapped in synchrony with a stimulus in either the auditory or the visual modality. Normally, this metronome stimulus is constant and various manipulations are performed outside the metronome itself, for example by requiring people to stop tapping at certain points (e.g., Castro-Meneses \& Sowman, 2018). Interestingly, previous research has indicated that even small perturbations in the timing of a metronome are reflected in synchronized tapping performance (Elliott, Welchman, \& Wing, 2009; Repp, 2000; Repp, 2001). Perturbations in a metronomic 500-ms timing stimulus as small as $4 \mathrm{~ms}$ (Repp, 2000) can immediately affect subsequent taps. ${ }^{1}$

Here, we asked participants to tap along with a metronome stimulus. We intermittently changed the metronome stimulus color (in the visual modality) or pitch (in the auditory modality), effectively inserting oddball stimuli. The timing of the oddball was consistent with the timing of the standard metronome stimulus. If an oddball is perceived to appear earlier than a non-oddball stimulus, then we expect an effect similar to what would occur if the oddball was genuinely shifted earlier - the subsequent taps would be earlier than otherwise (see Elliott et al., 2009). Thus, we hypothesize that on the tap following an oddball there will be a compensation for this change in perceived timing such that the timing of the next tap will be earlier than it would be otherwise. The tap occurring at the time of the oddball, in contrast, is not expected to be earlier. This is because tapping is predictive, occurring prior to

\footnotetext{
${ }^{1}$ Note, in these experiments, the small change in timing is carried over into a new timing of the metronome stimulus, such that if the metronome was at 500 $\mathrm{ms}$, and then a 496-ms metronome occurred, the following stimulus would still occur $500 \mathrm{~ms}$ after that small deviation, rather than say $504 \mathrm{~ms}$ (which would compensate for the change).
}

the metronome stimulus presentation (see Repp, 2005, for review). Even if the oddball was perceived much earlier than the standard, the time taken to "react" to this perceived earlier onset would likely be later (see the discussion of RTs above). Further, because the metronome stimulus returns to a standard immediately after the oddball, the timing of the tap following this return to the standard stimulus should also return towards the previous tap timing.

\section{Experiment 1: Oddball reactions}

\section{Participants}

Forty participants took part in this experiment - mean age $=$ 20.9 years, SD $=9.7$ years, four left-handed, 12 male. Responses were collected using the same method as taps in Experiment 1. Participants were told to respond as quickly as possible to the target stimuli (whether oddball or standard).

All participants in this experiment, and all subsequent experiments, were gathered from the psychology participant pool. Participants received course credit for participation. Participants provided written consent in accordance with the Declaration of Helsinki. The experiment was approved by the Macquarie University Ethics Committee.

\section{Equipment}

Experimental stimuli were presented on an AOC G2770PF (27-in) monitor controlled by a Dell Optiplex 9010 PC (8GB RAM, 3.2 Ghz Intel i5-3470 CPU) running 64-bit Windows 7. Neurobehavioral System's Presentation (v20.2) was used to present stimuli and record responses. Tasks took place in dimly lit, sound-dampened rooms, with participants seated $0.8 \mathrm{~m}$ away from their monitor, and speakers placed 0.7 $\mathrm{m}$ away from participants for binaural sound presentation. Responses were collected on a Cedrus RB-840 button box and were performed with the index finger of the dominant hand. All experiments used the same equipment.

\section{Stimuli and procedure}

Visual stimuli were grey and blue circles (125-pixel circumference; RGB: $128 ; 128 ; 128$ and 0; 0; 200, respectively) and auditory stimuli were high-pitched and low-pitched tones $(\approx$ $80 \mathrm{db}, 1,200 \mathrm{~Hz}$ and $800 \mathrm{~Hz}$, respectively). Again, these stimuli were consistent across all experiments.

Participants completed four blocks of 70 trials. Two blocks were responding to visual stimuli and two to auditory stimuli. These blocks were presented in order, either all auditory or all visual, and which set of blocks were presented first was counterbalanced. Further, which stimulus was the standard and which was the oddball was also counterbalanced. In this 
experiment, between every sixth and tenth response imperative was an oddball, randomly selected from a uniform distribution.

Each trial was initiated with a fixation cross, presented centrally for $500 \mathrm{~ms}$, followed by a blank screen lasting between $500 \mathrm{~ms}$ and $1,000 \mathrm{~ms}$ randomly selected for each trial from a uniform distribution. The response imperative was then presented for $100 \mathrm{~ms}$ followed by a blank screen until the participant responded. A 300-ms blank screen was then presented prior to the presentation of the next trial.

\section{Analysis}

The first three trials of each block were discarded. Further, any trials with RTs slower than 1,500 ms and RTs faster than 100 $\mathrm{ms}^{2}$ were discarded as lapses in attention and pre-emptive responses, respectively (3.5\% of responses). Mean RTs were calculated for three conditions, two standards in a row (S-S), a standard and then an oddball (S-O) and an oddball then a standard (O-S). The coefficients of variation (CVs) were also calculated as the standard deviation of RTs divided by the mean RTs. This is a measure of response variability. An ANOVA was performed with the modality of the stimulus and the oddball status as within-subject factors on both the mean RTs and CVs. The order the participants performed the task in (visual then auditory or vice versa) and which stimulus was an oddball and which was a standard did not make a significant difference when included as betweensubjects factors, and therefore the simpler ANOVA is reported here.

For this, and all subsequent experiments, GreenhouseGiesser (GG) corrections for violations of sphericity were applied. Follow-up analysis was done using t-tests with the Holm correction (Holm, 1979). Partial eta squared $\left(\eta_{p}{ }^{2}\right)$ is reported as a measure of effect size for ANOVA results, and Cohen's $\mathrm{d}(d)$ is reported for t-tests.

\section{Results}

\section{Mean reaction times (RTs)}

Mean RTs were $225 \mathrm{~ms}$. There was no main effect of the modality of the target stimulus $(\mathrm{F}(1,39)=1.30, \mathrm{p}=.261$, $\left.\eta_{\mathrm{p}}{ }^{2}=.03\right)$. The main effect of the sequence of prior and current imperatives was significant $\left(\mathrm{F}(2,78)=7.49, \mathrm{p}=.001, \eta_{\mathrm{p}}{ }^{2}=\right.$ .16). Given an S-S combination of targets, RTs were $222 \mathrm{~ms}$, while RTs were marginally slower to an O-S combination $(224 \mathrm{~ms})$ and slowest given an S-O combination $(231 \mathrm{~ms})$. However, this main effect was superseded by the interaction effect $\left(F(2,78)=4.61, p=.013, \eta_{p}{ }^{2}=.10\right.$, Fig. 2, left $)$, and therefore was not analyzed further.

\footnotetext{
${ }^{2}$ Note, adjusting these limits did not significantly alter results.
}

To analyze this interaction, we compared RTs between each combination of stimuli separately in the visual and auditory modality. In the auditory modality, each combination of trials was significantly different to the others; S-S RTs (226 $\mathrm{ms})$ were significantly shorter than O-S RTs $(235 \mathrm{~ms}$; $\mathrm{t}(39)=$ $2.41, \mathrm{p}=.041$, Holm-corrected, $d=.10)$ and S-O RTs (238 $\mathrm{ms} ; \mathrm{t}(39)=3.86, \mathrm{p}=.001$, Holm-corrected, $d=.18$ ). The RTs to O-S stimuli were significantly shorter than to S-O stimuli $(\mathrm{t}(39)=2.05, \mathrm{p}=.048$, Holm-corrected, $d=.09)$.

In the visual modality, there was no significant difference in RTs between the different conditions, S-S RTs were 215 $\mathrm{ms}$, O-S RTs were $216 \mathrm{~ms}$, and S-O RTs were $219 \mathrm{~ms}$ (S-S compared to O-S; $\mathrm{t}(39)=1.11, \mathrm{p}=.617$, Holm-corrected, $d=$ $.15 \mathrm{~S}-\mathrm{S}$ compared to S-O; $\mathrm{t}(39)=.576, \mathrm{p}=.617$, Holmcorrected, $d=.07$; S-O compared to O-S; $\mathrm{t}(39)=1.29, \mathrm{p}=$ .617 , Holm-corrected, $d=.18$ ).

\section{Coefficients of variation (CVs)}

The mean CV was .267. CVs were not significantly affected by whether the target stimulus was auditory or visual $(\mathrm{F}(1,39)$ $\left.=.222, \mathrm{p}=.640, \eta_{\mathrm{p}}{ }^{2}=.01\right)$, nor by the interaction between modality and the prior to current combination of targets $(\mathrm{F}(2$, 78) $=2.01, \mathrm{p}=.141, \eta_{\mathrm{p}}{ }^{2}=.05$ ). There was a main effect of the prior to current target combination $(\mathrm{F} 2,78)=3.50, \mathrm{p}=.043$, GG-corrected, $\eta_{\mathrm{p}}{ }^{2}=.08$, Fig. 2, right).

Post hoc analysis showed that CVs were significantly lower following an O-S combination (.239) compared to both an S-S combination $(.283 ; \mathrm{t}(39)=2.82, \mathrm{p}=.023$, Holmcorrected, $d=.44)$ and an S-O combination $(.279 ; \mathrm{t}(39)=$ 2.34, $\mathrm{p}=.049$, Holm-corrected, $d=.39$ ). The S-S and S-O combinations did not result in significantly different CVs $(\mathrm{t}(39)=.209, \mathrm{p}=.835$, Holm-corrected, $d=.04)$.

\section{Discussion}

In this experiment, participants were required to react as quickly as possible to any given stimulus. For half of the blocks, reactions were to visual stimuli, while for the other half reactions were to auditory stimuli. Occasionally, the standard stimulus was replaced by an oddball stimulus. Interestingly, while in the visual modality no effects were found, in the auditory modality, the opposite pattern to what was expected emerged RTs were slowest given an S-O combination of trials. Further, an O-S combination resulted in the second slowest RTs, and S$\mathrm{S}$ trials were responded to quickest.

The current findings in the auditory modality are consistent with an oddball being perceived to onset slightly later than the standard stimulus. When an oddball was presented in the current trial, RTs were slowest, consistent with the oddball stimulus being perceived later than a standard stimulus. As for why an O-S combination would also be responded to objectively slower than an S-S combination, perhaps this can be 

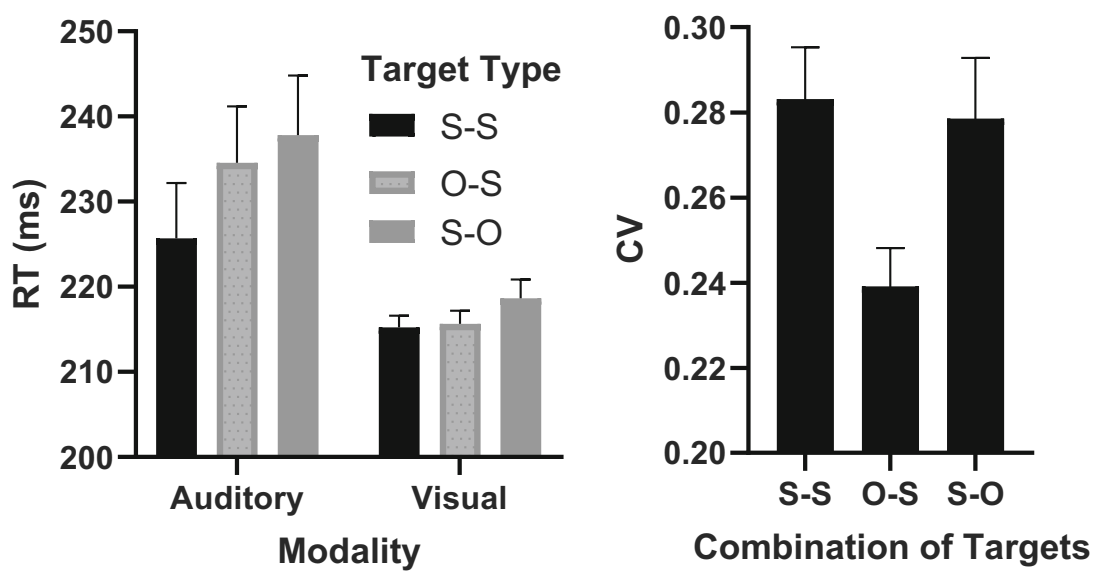

Fig. 2 Left: Reaction times (RTs) in each modality to each combination of prior to current target type. Right: Coefficients of variation (CVs) across each combination of prior and current target type. Error bars represent one standard error of the mean

attributed to some form of cost associated with the presentation of an oddball. For example, the lower RTs to an oddball could lead to slower subsequent RTs due to a "response refractory period" (Vallesi, McIntosh, \& Stuss, 2009; Vallesi, Mussoni, et al., 2007; Vallesi \& Shallice, 2007; Vallesi, Shallice, \& Walsh, 2007). Interestingly, O-S combinations were also reacted to with less variability than to the other two combinations. Why this should be is not known, but perhaps the presentation of an oddball stimulus grabbed attention (Tse, 2010; Tse et al., 2004), resulting in less RT variability in the following trial.

However, in the visual modality, RTs were not significantly affected by the combination of prior to current target stimuli. One reason for this difference is that perhaps the presentation of an oddball sound was more stimulating than an oddball visual stimulus. While a simple change in color has been shown to elicit the oddball duration illusion (Wehrman, 2020a; Wehrman et al., 2018a, b), perhaps in terms of RTs the deviance was not large enough to result in an effect. In future, perhaps the relative "oddness" between visual and auditory modalities requires calibration, though given previous oddball duration illusion effects, a result should have been expected here if present.

Kim and McAuley (2013) and Matthews (2015) have previously attempted RT tasks in relationship to oddball stimuli. Kim and McAuley (2013) required participants to respond to auditory oddballs that were either close or far in pitch from a standard oddball. Generally, Kim and McAuley (2013) found that RTs were faster to further pitched oddball stimuli (though primarily when oddballs were increasing in pitch, rather than decreasing). While this is in the opposite direction to the findings here, because Kim and McAuley (2013) did not compare RTs to standard stimuli (as well as rarity not convincingly affecting RTs), it is difficult to judge the gating of oddballs from their findings alone.

Compounding this, in Matthews (2015), participants were required to categorize the gender of faces, finding that when a face repeated, RTs were shorter than if the face did not repeat. ${ }^{3}$ In this regard, the current experiment further exemplified this finding; even when not requiring a judgment, repetition led to comparatively shorter RTs compared to non-repeated (but still, relative to the Matthews (2015) article, not rare) stimuli. However, it is noteworthy that in another experiment from the same article, Matthews (2015) found that simply detecting the onset of a non-face stimuli (without categorization) did not lead to different RTs depending on stimulus repetition or repetition rate. One possible reason for this difference is methodological. In the current article, and the other experiments mentioned using RTs, a response was required on every trial. Contrastingly, in the onset-detection task from Matthews (2015), catch trials in which a response imperative was not shown were included. This requirement to possibly inhibit a response likely slowed all RTs (e.g., Buckolz \& Rodgers, 1980), possibly diminishing any difference from repetition.

As an alternative possibility, perhaps, given an oddball stimulus in the auditory modality, RTs were longer due to a delay in response, rather than a delay in perception. For example, it has been shown that oddballs can limit attentional resources (Asplund, Todd, Snyder, Gilbert, \& Marois, 2010) and thus perhaps affect RTs. Therefore, in Experiment 2, we removed the RT component of the task by asking participants to produce a specific duration rather than react to a stimulus. In this second experiment, participant productions were automatically initiated by either an oddball or a standard stimulus. Only data from the visual modality were collected due to the (COVID-19 related) cessation of in-person data collection and thus unavailability of the same equipment used for the other experiments.

\footnotetext{
${ }^{3}$ Note, this effect was not present if the face was not repeated, but the gender was the same, ruling out response bias.
} 


\section{Experiment 2: Oddball productions}

\section{Participants}

Twenty participants took part in this experiment - mean age $=$ 23.2 years, SD $=10.1$ years, five left-handed, five male. Participants were told to produce durations as accurately as possible without counting.

\section{Stimuli and procedure}

The stimuli used were as per the visual stimuli in Experiment 1 , and which stimulus was the oddball was counterbalanced between subjects. Participants completed four blocks of 50 trials. Each block required the production of a different duration: $500 \mathrm{~ms}, 750 \mathrm{~ms}, 1,000 \mathrm{~ms}$, and 1,250 ms. Participants were told the target duration before the start of each block. The order of these blocks was randomized. In this experiment, between every third and sixth stimulus initiating a production was an oddball, randomly selected from a uniform distribution.

Each trial was initiated with a fixation cross, presented centrally for $500 \mathrm{~ms}$. This was followed by a blank screen lasting between $500 \mathrm{~ms}$ and 1,000 ms, randomly selected each trial from a uniform distribution. The stimulus initiating the participant production was then presented for $50 \mathrm{~ms},{ }^{4}$ followed by a blank screen until the participant terminated the production. A blank screen was presented for $300 \mathrm{~ms}$ followed by a screen showing the duration produced by the participant for $500 \mathrm{~ms}$, allowing the participant to adjust their productions in the following trials. Another 300-ms blank screen preceded the start of the next trial.

\section{Analysis}

The first three trials of each block were discarded. Further, any trials with productions shorter than $200 \mathrm{~ms}$ and longer than 4,000 ms were discarded as reactions to the onset of the stimulus and lapses of attention, respectively (1.7\% of productions). Mean productions were calculated for both oddball or standard stimuli at each produced duration. Further, the CVs were also calculated as the standard deviation of productions divided by the mean production. An ANOVA was performed with the oddball status and the requested production duration as within-subject factors.

\footnotetext{
${ }^{4}$ A shorter duration was used here to minimize any possible clock effects that could have extended productions, a feature that was not of concern in Experiments 1 and 2 .
}

\section{Results}

\section{Productions}

The mean produced duration was $906 \mathrm{~ms}$. There was a main effect of the duration requested for production $(\mathrm{F}(3,57)=$ 13.87, $\mathrm{p}<.001$, GG-corrected, $\left.\eta_{\mathrm{p}}{ }^{2}=.42\right)$. The mean productions for each requested duration was as expected $(500 \mathrm{~ms}$ requested $=617 \mathrm{~ms} ; 750 \mathrm{~ms}=774 \mathrm{~ms}, 1,000 \mathrm{~ms}=1,023 \mathrm{~ms}$, and $1,250 \mathrm{~ms}=1,203 \mathrm{~ms}$ ) and were not analyzed further. Further, there was a main effect of whether a production was initiated with an oddball or a standard stimulus $(\mathrm{F}(1,19)=5.11, \mathrm{p}=$ $.036, \eta_{\mathrm{p}}{ }^{2}=.21$, Fig. 3 , Left). This effect showed that productions were longer following an oddball $(921 \mathrm{~ms})$ rather than a standard stimulus (902 ms). The interaction was not significant $\left(\mathrm{F}(3,57)=1.89, \mathrm{p}=.174\right.$, GG-corrected, $\left.\eta_{\mathrm{p}}{ }^{2}=.09\right)$.

\section{CVs}

The mean CV was .235. The main effect of requested duration was not significant after correction for sphericity $(F(3,57)=$ $3.04, \mathrm{p}=.056$, GG-corrected, $\left.\eta_{\mathrm{p}}{ }^{2}=.14\right)$. The main effect of the type of stimulus initiating the production was significant $(\mathrm{F}(1$, 19) $=9.98, \mathrm{p}=.005, \eta_{\mathrm{p}}{ }^{2}=.34$, Fig. 3, Right), showing that $\mathrm{CVs}$ were higher given a standard stimulus $(.250)$ rather than an oddball stimulus (.220). The interaction effect was not significant $\left(\mathrm{F}(3,57)=.105, \mathrm{p}=.886, \mathrm{GG}\right.$-corrected, $\left.\eta_{\mathrm{p}}{ }^{2}=.01\right)$.

\section{Discussion}

In Experiment 2, participants were required to produce a given duration by terminating an interval automatically initiated by either an oddball or a standard stimulus. We hypothesized that if an oddball was perceived to begin earlier, then productions should be shorter given an oddball stimulus. However, we found the opposite effect; productions were longer given an
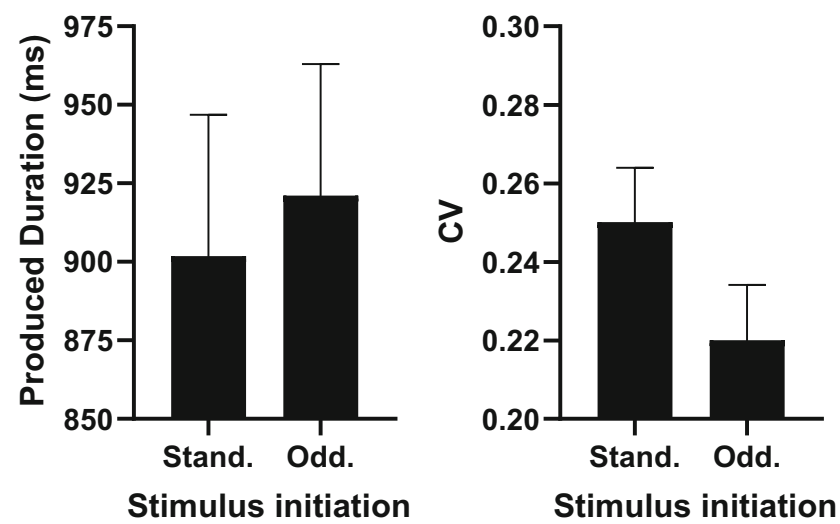

Fig. 3 Left: Mean produced durations depending on the type of stimulus initiating the production. Right: Coefficients of variation (CVs) as depending on the type of stimulus initiating the production. Error bars represent one standard error of the mean 
oddball stimulus. This could indicate that an oddball was perceived to start later than a standard stimulus, or that the oddball stimulus otherwise interfered with the initiating of timing.

Interestingly, initiating a production with an oddball resulted in slightly less variable productions compared to when initiated with a standard. Such an effect could be attributed to the consistency with which the stimulus onset was gated. If true, this would indicate that while an oddball is gated slightly later than a standard stimulus, it is also more reliably gated than a standard stimulus. Again, perhaps this could be attributed to the attentional effects proposed to underly the oddball duration illusion (Tse, 2010; Tse et al., 2004). Notably, we provided feedback on each trial in this experiment, allowing participants to adjust their performance from one trial to the next in order to more accurately produce the durations. Providing feedback has been shown to reduce variability across multiple time perception tasks, including duration production (Droit-Volet \& Izaute, 2005; Saito, Janssen, \& Tayama, 2015; Wearden \& Farrar, 2007). Thus, any effect of variability found here is likely to be smaller than if feedback was not present, though this requires empirical investigation.

Finally, it is worth noting the possibility that an oddball sped up the perceived passage of time. This is the common explanation of the oddball duration illusion, and could be achieved by the presentation of an oddball speeding up some internal "pacemaker," resulting in more perceived time accumulating over a given amount of objective time, as per the scalar expectancy theory (SET; see Gibbon, Church, \& Meck, 1984; Wearden, 1991; Wearden \& Lejeune, 2008). Firstly, if the presentation of an oddball sped up the perceived passage of time, then duration production should have been shorter, rather than longer. Therefore, the current experiment provides evidence directly counter to this possibility. If an oddball does increase the perceived passage time, then this is likely to occur only while an oddball is presented (i.e., continuously on screen). Alternatively, it is possible that the oddball duration illusion arises purely in memory, such that an interval is remembered as longer due to the presence of an oddball stimulus (see Matthews \& Gheorghiu, 2016). In addition, if there was any type of pacemaker effect (e.g., a slowing of perceived time by the initiating of an interval with an oddball), then an interaction effect should have been present between the duration produced and whether the production was initiated with a standard or oddball. Such an effect has been reported, for example, in differences between the perceived passage of time for sounds versus lights (Wearden, Edwards, Fakhri, \& Percival, 1998); as the duration estimated increases, the differences between intervals represented by lights and sounds increased. However, again, such an effect was not present here. Therefore, the current experiment seems to indicate that, at least when initiating a timed interval, the presentation of an oddball does not have a pacemaker-based effect on perceived time.

\section{Experiment 3: Tapping oddball}

\section{Methods}

\section{Participants}

Sixty participants took part in this experiment, 30 performed tapping in the visual condition (mean age $=20.5$ years, $\mathrm{SD}=$ 6.1 years, three left-handed, nine male) and 30 in the auditory condition (mean age $=20.1$ years, $\mathrm{SD}=4.1$ years, one lefthanded, 11 male).

\section{Stimuli and procedure}

The stimuli used were as per Experiment 1. Which stimulus was used as a standard and which was an oddball was counterbalanced between participants (15 participants per counterbalanced group). The stimulus onset asynchrony (SOA) measured from the initiation of one stimulus to the initiation of the next was $600 \mathrm{~ms}$. Stimulus duration was $100 \mathrm{~ms}$.

Participants were exposed to an initial training block of 20 taps in which the experimenter tapped for the first five stimuli, and the participant then took over. Participants were informed that occasionally the stimulus (either auditory or visual, depending on the group), would be different to the normal, but to keep tapping in synchrony despite the change.

Participants then performed three blocks of 80 beats, with self-paced breaks between. An oddball stimulus replaced the standard stimulus between four and eight beats, randomly chosen for each oddball presentation using a uniform distribution.

\section{Analysis}

Our analysis focused on four taps - the tap immediately prior to the oddball, the oddball tap, and the subsequent two oddball taps. We initially grouped all stimuli into sets of four taps (prior, current, subsequent one, and subsequent two). The first five taps of each block were discarded. Further, because taps were analyzed in sets of four, if a non-oddball stimulus had an oddball in any of the other four positions (i.e., just before it, or up to two stimuli after it) then that standard was not included in the analysis. This was to prevent "double counting" of stimuli in both the standard and oddball conditions. Finally, taps outside of $300 \mathrm{~ms}$ in either direction of the stimulus were discarded. Overall, $6.7 \%$ of trials were discarded in the auditory condition and $13.7 \%$ of trials in the visual condition. For each participant, we next averaged together the asynchrony of the taps to the standard stimuli and subtracted them from the asynchrony of the four taps related to the oddball. Essentially, this gave us a measure of how much the oddball stimulusrelated taps differed from the timing of the taps related to standard stimuli. 
Asynchronies were used as the dependent variable in an ANOVA with the within-subject factor of which tap was examined $(\mathrm{O}-1, \mathrm{O}, \mathrm{O}+1$ or $\mathrm{O}+2$ for the tap prior to the oddball, the tap to the oddball, the tap after the oddball or the second tap after the oddball, respectively). Two between-subject factors were included - whether participants tapped to visual or auditory stimuli, and, as a control, which counterbalance group the participant was in.

\section{Results}

In the visual modality, the mean tap in synchrony to a standard stimulus was $68.8 \mathrm{~ms}(\mathrm{SD}=44.3 \mathrm{~ms})$ prior to the actual stimulus occurring, while the oddball was tapped to at $62.4 \mathrm{~ms}(\mathrm{SD}=119.1 \mathrm{~ms})$ prior to the stimulus. In the auditory modality, the mean asynchrony of a tap to a standard stimulus was $60.6 \mathrm{~ms}(\mathrm{SD}=47.9 \mathrm{~ms})$ prior to the actual stimulus occurring, while the oddball related taps were on average $55.6 \mathrm{~ms}(\mathrm{SD}=67.0 \mathrm{~ms})$ prior to the stimulus.

There was no main effect of which counterbalanced group the subject participated in $\left(\mathrm{F}(1,56)=.475, \mathrm{p}=.494, \eta_{\mathrm{p}}{ }^{2}=\right.$ $.01)$. There was a main effect of whether stimuli were auditory or visual $\left(\mathrm{F}(1,56)=4.09, \mathrm{p}=.048, \eta_{\mathrm{p}}{ }^{2}=.07\right)$, showing that the mean of the four taps around the oddball in the auditory domain was $0.1 \mathrm{~ms}$ earlier than the taps to the auditory standard stimulus while the mean of the four taps around the oddball in the visual domain was at $6.9 \mathrm{~ms}$ earlier than the taps to the visual standard stimulus. The main effect of which of the four taps was examined was significant $(F(3,168)=$ $6.86, \mathrm{p}=.003$, GG-corrected, $\left.\eta_{\mathrm{p}}{ }^{2}=.11\right)$. The tap before the oddball (O-1) was tapped at $5.3 \mathrm{~ms}$ earlier than the tap to a standard, while the oddball tap $(\mathrm{O})$ was tapped at $8.2 \mathrm{~ms}$ earlier than the standard taps. The tap after the oddball (O-1) was at $12.0 \mathrm{~ms}$ earlier compared to the standards, while the next tap (O-2) was at $0.9 \mathrm{~ms}$ later compared to the standards. However, this effect was superseded by an interaction and not analyzed further.

The interaction between counterbalanced group with both the modality $\left(\mathrm{F}(1,56)=.245, \mathrm{p}=.622, \eta_{\mathrm{p}}{ }^{2}<.01\right)$ and which tap was examined $(\mathrm{F}(3,168)=.088, \mathrm{p}=.890$, GG-corrected, $\left.\eta_{\mathrm{p}}{ }^{2}<.01\right)$ were not significant. The three-way interaction was also not significant $(\mathrm{F}(3,168)=.165, \mathrm{p}=.816$, GG-corrected, $\left.\eta_{\mathrm{p}}^{2}<.01\right)$.

The interaction between the modality of the stimulus and the tap in relation to the oddball was significant $(\mathrm{F}(3,168)=$ $4.58, \mathrm{p}=.017$, GG-corrected, $\eta_{\mathrm{p}}^{2}=.08$, Fig. 4). To further analyze this data, we examined whether each tap was significantly different to zero (i.e., the same timing as the mean standard tap).

In the auditory modality, the $\mathrm{O}-1$ tap was $1.2 \mathrm{~ms}$ later than the mean tap to a standard stimulus $(\mathrm{t}(29)=.324, \mathrm{p}=.979$, Holm-corrected, $d=.06$,), $\mathrm{O}+1$ tap was $2.9 \mathrm{~ms}$ earlier $(\mathrm{t}(29)=$ $.700, \mathrm{p}=.979$, Holm-corrected, $d=.13$ ), and the $\mathrm{O}+2$ tap was

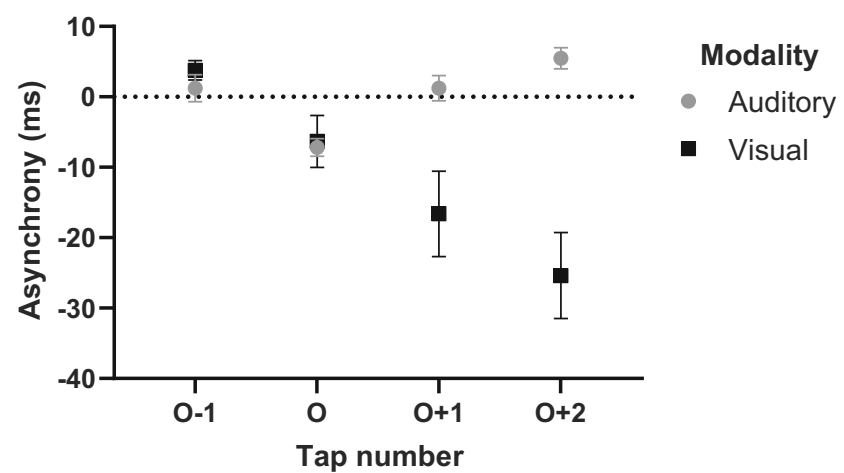

Fig. 4 Tapping asynchronies in both the visual and auditory modality, in which negative values indicate tapping earlier than the mean asynchrony to the standard stimulus. Asynchronies were calculated as the mean oddball tap asynchrony, less the mean standard tap asynchrony for each group. Error bars represent one standard error of the mean

$9.5 \mathrm{~ms}$ later $(\mathrm{t}(29)=2.44, \mathrm{p}=.063$, Holm-corrected, $d=.45)$; none of these were significantly different to zero, i.e., the timing of the average standard tap. The tap synchronous with the oddball stimulus, $\mathrm{O}$, was significantly earlier than the average standard tap (by $8.3 \mathrm{~ms}, \mathrm{t}(29)=2.90, \mathrm{p}=.023$, Holmcorrected, $d=.53$ ).

In the visual modality, $\mathrm{O}-1$ (later by $9.4 \mathrm{~ms}, \mathrm{t}(29)=.1 .73$, $\mathrm{p}$ $=.104$, Holm-corrected, $d=.32), \mathrm{O}($ earlier by $8.1 \mathrm{~ms}, \mathrm{t}(29)=$ $2.26, \mathrm{p}=.093$, Holm-corrected, $d=.41$ ), and $\mathrm{O}+2$ (earlier by $7.7 \mathrm{~ms}, \mathrm{t}(29)=2.03, \mathrm{p}=.104$, Holm-corrected, $d=.37$ ) were not significantly different to the timing of the average standard tap. However, tap O+1 (earlier by $11.1 \mathrm{~ms}, \mathrm{t}(29)=3.30, \mathrm{p}=$ .010 , Holm-corrected, $d=.60$ ) was significantly earlier than the average tap asynchrony to the standard stimulus.

\section{Discussion}

In this experiment, participants were asked to tap in synchrony with a metronome stimulus in either the visual or the auditory modality. Occasionally, an oddball stimulus was presented in place of the standard metronome stimulus. We theorized that if an oddball stimulus was perceived to begin earlier than a standard stimulus, then the tap after (i.e., to the next stimulus) the oddball stimulus should be relatively earlier. In the auditory modality, an oddball stimulus resulted in an earlier tap than a non-oddball stimulus to that stimulus. This did not conform to the hypothesis forwarded here. In the visual modality, however, the tap subsequent to the oddball was earlier than taps to a standard stimulus. This was in line with the hypothesized effect. The different results across the two modalities are discussed in turn.

The results in the auditory modality seems counterintuitive given that in tapping experiments, participants are expected to tap predictively (before) to the metronome stimulus. Given that the mean tap time was $60.6 \mathrm{~ms}$ earlier in the auditory domain in comparison to the metronome stimulus, it seems unlikely that participants were reactively responding 
earlier to the oddball stimulus. ${ }^{5}$ Thus, why participants tapped earlier to an oddball requires further research. One possibility is the gradual speeding up of taps in expectation to an oddball, resulting in an earlier oddball-linked tap. However, why this would occur in the auditory modality, and not (significantly at least; though numerically similar asynchronies) in the visual domain is unknown.

In the visual modality, participants did indeed tap earlier to the metronome stimulus immediately following the oddball. This is in line with an oddball being perceived as occurring earlier than a standard stimulus. This likely occurred in the visual modality and not the auditory modality due to the tighter locking of taps to auditory stimuli compared to visual stimuli (Jäncke, Loose, Lutz, Specht, \& Shah, 2000; Repp \& Penel, 2002; Repp \& Penel, 2004) and the tendency for tapping errors with auditory metronome stimuli to be corrected for more quickly (Kato \& Konishi, 2006).

\section{General discussion}

In the current research, we did not find consistent evidence that an oddball stimulus was perceived to onset earlier (i.e., was gated earlier) than a standard stimulus. In Experiment 3, there was earlier tapping in relation to the stimuli after an oddball, consistent with an oddball being gated earlier than a nonoddball stimulus. However, this only occurred in the visual modality. Further, the results of both Experiment 1 and Experiment 2 seem to indicate that an oddball stimulus is gated later than a standard stimulus. If indeed this is the case, the amount of time by which an oddball is perceived to onset later than a standard is fairly short. In Experiment 1 , specifically in the auditory modality, oddball stimuli were reacted to $\approx 16 \mathrm{~ms}$ later than a standard stimulus. Further, in Experiment 2, a visual oddball initiating the production resulted in $\mathrm{a} \approx 9 \mathrm{~ms}$ longer production compared to when initiated with a standard stimulus. In this regard, it is noteworthy that very short oddballs can be perceived to last shorter (rather than longer) than nonoddballs (see Tse et al., 2004; Ulrich, Nitschke, \& Rammsayer, 2006). Though we had envisioned the current task set as capturing an earlier gating of the oddball stimulus, perhaps it is instead the case that oddball stimuli are actually gated later than non-oddball stimuli, as found in Experiments 1 and 2. Perhaps, in this regard, a longer duration is required for the nononset-based timing effect to overcome the initially later gating of the oddball, allowing for the stereotypical oddball duration illusion.

\footnotetext{
${ }^{5}$ Note, a "surprise" effect of the oddball in the auditory modality resulting in earlier release of a response, such as can be seen in the start-react paradigm (Carlsen, Almeida, \& Franks, 2012; Castellote, Van den Berg, \& Valls-Sole, 2013; Valls-Solé et al., 1995; Valls-Solé, Kofler, Kumru, Castellote, \& Sanegre, 2005) cannot explain this effect, as taps were prior to the presentation of the oddball.
}

This raises the question of why we found earlier taps in Experiment 3 following an oddball in the visual modality. Though this requires further research, it is noteworthy that in electroencephalography studies, alpha-band synchronization is predictive of tapping performance (see Kawasaki, Kitajo, \& Yamaguchi, 2018), while oddball presentation causes broad cortical alpha-band desynchronization particularly in the visual modality (see Peng, Hu, Zhang, \& Hu, 2012). Taken together, perhaps it is the case that visual oddball presentation caused a desynchronization in alpha-band activity, which subsequently disturbed tapping performance. However, this is speculative, and requires further research to support.

Given how short the duration effects found in Experiments 1 and 2 were (9-16 ms), if there is a delay of gating for oddball stimuli, it is unlikely that tasks such as bisection would be affected by differences in onset timing. This is because, typically, the step-size of the durations are around $50 \mathrm{~ms}$ (Birngruber et al., 2014; Matthews, 2011; Matthews \& Gheorghiu, 2016; Wehrman, 2020a; Wehrman et al., 2018a, b). Further, in examining simultaneity judgments, the minimum step size was $35 \mathrm{~ms}$ in Wehrman (2020a), again longer than the gating effects found here. Instead, it seems likely that the oddball duration illusion, at least at longer target durations, arises from effects outside of onset.

As an interesting alternative, ${ }^{6}$ there is some evidence that an oddball may delay the detection of the offset of a stimulus. Particularly, if a stimulus grabs attention, such as an oddball is proposed to (see Tse et al., 2004), the perceived offset of that stimulus will be later (Rolke, Ulrich, \& Bausenhart, 2006). If this is the case, perhaps a contributing factor to the oddball duration illusion is a delay in the offset of the oddball stimulus, extending the perceived duration of the stimulus. In the current experiment set, particularly in Experiments 1 and 2, it also may be that this is the effect we are detecting; perhaps participants were responding to the offset, rather than onset, of the stimulus, resulting in the subsequent delays in response and production, respectively.

However, it is also possible that the tasks used here did not appropriately capture the oddball effect. Generally, in an oddball task, participants are required to judge a single stimulus in a line of other stimuli (e.g., Tse et al., 2004; Wehrman et al., 2018a, b), or compare one stimulus to another (e.g., Matthews, 2011, 2015). Here, in Experiments 1 and 2, a single stimulus was presented on each trial, and in Experiment 3, a continuous line of stimuli were used with no break. There is some evidence that the experiments here were appropriate to capture the oddball effect, however. Firstly, it is worth noting that oddballs have been shown to have an effect on electroencephalography even when continuous sets of stimuli are used (e.g., Barry, Kirkaikul, \& Hodder, 2000; Thee, Nisar, \& Soh, 2018). Further, Wehrman (2020a) demonstrated that the oddball duration illusion could be found even when every trial is

\footnotetext{
${ }^{6}$ Credit to Reviewer \#1, as we had not considered this possibility.
} 
judged and the target stimulus was either rare or standard. Thus, the conditions used here have previously been shown to be able to elicit standard oddball effects.

It is worth noting a caveat of the above experiments. The oddball duration illusion tends to arise due to a sequence of events which are at least somewhat time sensitive. For example, Matthews (2015) found that if the time between a standard and a second image exceeded 2,000 ms, then the oddball duration illusion was eliminated. Indeed, in electroencephalography experiments, extended interstimulus intervals reduce the neural response to oddballs (e.g., Kurkela, Lipponen, Kyläheiko, \& Astikainen, 2018). While here we used rarity (rather than direct repetition or change), it is quite possible that the durations between subsequent stimuli in Experiments 1 and 2 resulted in a reduction of the oddball effect. Instead, perhaps if an oddball duration illusion is induced purely by rarity, it may require higher level mediation than would be expected to be present in the gating of a stimulus (i.e., a participant would have to think about the duration of the oddball, rather than simply reacting to it). In this regard, however, it is interesting that we found an effect of an oddball at all in Experiments 1 and 2 where the duration between presentations of subsequent stimuli were extended.

\section{Future studies}

There are numerous possible studies following on from the current research, aside from those mentioned above. One particularly fruitful approach to examining the slower than expected RTs and productions when presented with an oddball stimulus may be the use of electroencephalography. There are numerous neural correlates of oddball presentation (e.g., the P300 and Mismatched Negativity; see Bénar et al., 2007; Garrido, Kilner, Stephan, \& Friston, 2009; respectively). It would be interesting to correlate RTs and productions with specific oddball-induced electroencephalographic components. If there is indeed later gating of an oddball stimulus, then we would expect latencies to be increased in those trials with later RTs and productions (see Walsh, Gunzelmann, \& Anderson, 2017, for the application of a similar idea).

Another possibility is the extension of the tapping experiment by combining the method used by Repp (2000) with that currently used here. Instead of comparing the taps in relation to the standard with the taps to the oddball, it could be useful to occasionally shift the timing of the oddball and standard stimuli. This would likely cause a perturbation in the following tap, which in turn could be compared between oddball and standard stimuli. A difference between these tap timings may be more telling as to how much, and why, a shift of taps following the oddball occurred in the tapping experiment here. ${ }^{7}$

\footnotetext{
${ }^{7}$ We thank Reviewer \# 3 for this idea.
}

\section{Conclusion}

In the current article, we have presented three experiments aimed at looking for evidence of early gating of oddball stimuli compared to non-oddball stimuli. In Experiment 3, the visual modality presented evidence in line with such an interpretation; however, Experiments 1 and 2 both presented evidence in the opposite direction. In sum, it seems unlikely that an oddball is gated earlier than a non-oddball stimulus as a consistent, modality-independent effect would have been expected. Instead, it seems likely that the primary driver of the oddball duration illusion is an expansion of perceived duration in some way. However, while we used tapping, RTs, and duration production, and Wehrman (2020a) used perceived simultaneity, it is still possible that perhaps other designs may find such an effect.

\section{References}

Alards-Tomalin, D., Leboe-McGowan, J. P., Shaw, J. D., \& LeboeMcGowan, L. C. (2014). The effects of numerical magnitude, size, and color saturation on perceived interval duration. Journal of Experimental Psychology: Learning, Memory, and Cognition, 40(2), 555.

Alexander, J. E., Porjesz, B., Bauer, L. O., Kuperman, S., Morzorati, S., O'CONNOR, S. J., .... Polich, J. (1995). P300 hemispheric amplitude asymmetries from a visual oddball task. Psychophysiology, 32(5), 467-475.

Alexander, P., Schlegel, A., Sinnott-Armstrong, W., Roskies, A. L., Wheatley, T., \& Tse, P. U. (2016). Readiness potentials driven by non-motoric processes. Consciousness and Cognition, 39, 38-47.

Asplund, C. L., Todd, J. J., Snyder, A. P., Gilbert, C. M., \& Marois, R. (2010). Surprise-induced blindness: a stimulus-driven attentional limit to conscious perception. Journal of Experimental Psychology. Human perception and performance, 36(6), 13721381. https://doi.org/10.1037/a0020551

Barry, R. J., Kirkaikul, S., \& Hodder, D. J. I. J. O. P. (2000). EEG alpha activity and the ERP to target stimuli in an auditory oddball paradigm. International Journal of Psychophysiology, 39(1), 39-50.

Bénar, C.-G., Schön, D., Grimault, S., Nazarian, B., Burle, B., Roth, M., ... Anton, J.-L. (2007). Single-trial analysis of oddball event-related potentials in simultaneous EEG-fMRI. Human Brain Mapping, 28(7), 602-613. https://doi.org/10.1002/hbm.20289

Birngruber, T., Schröter, H., \& Ulrich, R. (2014). Duration perception of visual and auditory oddball stimuli: Does judgment task modulate the temporal oddball effect? Attention, Perception, \& Psychophysics, 76(3), 814-828.

Buckolz, E., \& Rodgers, R. (1980). The influence of catch trial frequency on simple reaction time. Acta Psychologica, 44(2), 191-200. https:// doi.org/10.1016/0001-6918(80)90067-0

Cai, Z. G., \& Connell, L. (2016). On magnitudes in memory: An internal clock account of space-time interaction. Acta Psychologica, 168, 111. https://doi.org/10.1016/j.actpsy.2016.04.003

Carlsen, A. N., Almeida, Q. J., \& Franks, I. M. (2012). Startle decreases reaction time to active inhibition. Experimentalis Brain Research, 217(1), 7-14. https://doi.org/10.1007/s00221-011-2964-9 
Castellote, J. M., Van den Berg, M. E., \& Valls-Sole, J. (2013). The StartReact effect on self-initiated movements. Biomedical Research International, 2013, 471792. https://doi.org/10.1155/ 2013/471792

Castro-Meneses, L. J., \& Sowman, P. F. J. P. (2018). Stop signals delay synchrony more for finger tapping than vocalization: a dual modality study of rhythmic synchronization in the stop signal task. 6 , e5242.

Droit-Volet, S., \& Izaute, M. (2005). The effect of feedback on timing in children and adults: The temporal generalization task. The Quarterly Journal of Experimental Psychology Section A, 58(3), 507-520. https://doi.org/10.1080/02724980443000025

Eagleman, D., \& Pariyadath, V. (2009). Is subjective duration a signature of coding efficiency? Philosophical Transactions of the Royal Society of London B: Biological Sciences, 364(1525), 1841-1851.

Elliott, M. T., Welchman, A. E., \& Wing, A. M. (2009). MatTAP: A MATLAB toolbox for the control and analysis of movement synchronisation experiments. Journal of Neuroscience Methods, 177(1), 250-257. https://doi.org/10.1016/j.jneumeth.2008.10.002

Fromboluti, E. K., Jones, K. B., \& McAuley, J. D. (2013). Temporal preparation contributes to the overestimation of duration of 'oddball' events. Frontiers in Human Neuroscience Conference Absract: 14th Rhythm Production and Perception Workshop Birmingham. https:// doi.org/10.3389/conf.fnhum.2013.214.00013

Garrido, M. I., Kilner, J. M., Stephan, K. E., \& Friston, K. J. (2009). The mismatch negativity: a review of underlying mechanisms. Clinical Neurophysiology, 120(3), 453-463.

Gibbon, J., Church, R. M., \& Meck, W. H. (1984). Scalar timing in memory Annals of the New York Acadamy of Sciences, 423(1), 52-77.

Grondin, S., \& Rammsayer, T. (2003). Variable foreperiods and temporal discrimination. The Quarterly Journal of Experimental Psychology A, 56(4), 731-765. https://doi.org/10.1080/02724980244000611

Holm, S. (1979). A simple sequentially rejective multiple test procedure. Scandinavian journal of statistics, 65-70.

Jäncke, L., Loose, R., Lutz, K., Specht, K., \& Shah, N. J. (2000). Cortical activations during paced finger-tapping applying visual and auditory pacing stimuli. Cognitive Brain Research, 10(1), 51-66. https://doi. org/10.1016/S0926-6410(00)00022-7

Kato, M., \& Konishi, Y. (2006). Auditory dominance in the error correction process: A synchronized tapping study. Brain Res, 1084(1), 115-122. https://doi.org/10.1016/j.brainres.2006.02.019

Kawasaki, M., Kitajo, K., \& Yamaguchi, Y. (2018). Sensory-motor synchronization in the brain corresponds to behavioral synchronization between individuals. Neuropsychologia, 119, 59-67. https://doi.org/ 10.1016/j.neuropsychologia.2018.07.026

Kim, E., \& McAuley, J. D. (2013). Effects of pitch distance and likelihood on the perceived duration of deviant auditory events. Attention, Perception, \& Psychophysics, 75(7), 1547-1558. https://doi.org/10. 3758/s13414-013-0490-5

Kurkela, J. L. O., Lipponen, A., Kyläheiko, I., \& Astikainen, P. (2018). Electrophysiological evidence of memory-based detection of auditory regularity violations in anesthetized mice. Scientific reports, 8(1), 3027. https://doi.org/10.1038/s41598-018-21411-z

Lin, Y.-J., \& Shimojo, S. (2017). Triple dissociation of duration perception regulating mechanisms: Top-down attention is inherent. PLoS One, 12(8), e 0182639.

Los, S. A. (2010). Foreperiod and the sequential effect: Theory and data. In A. C. Nobre \& J. T. Coull (Eds.), Attention and time (pp. 289302). Oxford: Oxford University Press.

Matthews, W. J. (2011). Stimulus Repetition and the Perception of Time: The Effects of Prior Exposure on Temporal Discrimination, Judgment, and Production. PLoS One, 6(5), e19815. https://doi. org/10.1371/journal.pone.0019815
Matthews, W. J. (2015). Time perception: The surprising effects of surprising stimuli. Journal of Experimental Psychology: General, 144(1), 172.

Matthews, W. J., \& Gheorghiu, A. I. (2016). Repetition, expectation, and the perception of time. Current Opinion in Behavioral Sciences, 8 , 110-116. https://doi.org/10.1016/j.cobeha.2016.02.019

McAuley, J. D., \& Fromboluti, E. K. (2014). Attentional entrainment and perceived event duration. Philosophical Transactions of the Royal Society B: Biological Sciences, 369(1658). https://doi.org/10.1098/ rstb.2013.0401

Pariyadath, V., \& Eagleman, D. (2007). The effect of predictability on subjective duration. PLoS One, 2(11), e1264.

Peng, W., Hu, L., Zhang, Z., \& Hu, Y. (2012). Causality in the Association between P300 and Alpha Event-Related Desynchronization. PLoS One, 7(4), e34163. https://doi.org/10. 1371/journal.pone.0034163

Penney, T. B. (2003). Attention, Clock Speed, and Memory.

Rammsayer, T. H., \& Verner, M. (2014). The effect of nontemporal stimulus size on perceived duration as assessed by the method of reproduction. Journal of Vision, 14(5), 17-17. https://doi.org/10. $1167 / 14.5 .17$

Repp, B. H. (2000). Compensation for subliminal timing perturbations in perceptual-motor synchronization. Psychological Research, 63(2), 106-128. https://doi.org/10.1007/PL00008170

Repp, B. H. (2001). Phase correction, phase resetting, and phase shifts after subliminal timing perturbations in sensorimotor synchronization. Journal of Experimental Psychology: Human Perception and Performance, 27(3), 600.

Repp, B. H., \& Penel, A. (2002). Auditory dominance in temporal processing: New evidence from synchronization with simultaneous visual and auditory sequences. Journal of Experimental Psychology: Human Perception and Performance, 28(5), 1085-1099. https://doi. org/10.1037/0096-1523.28.5.1085

Repp, B. H., \& Penel, A. J. P. r. (2004). Rhythmic movement is attracted more strongly to auditory than to visual rhythms. 68(4), 252-270.

Repp, B. H. (2005). Sensorimotor synchronization: A review of the tapping literature. Psychonomic Bulletin and Review, 12, 969-992.

Rolke, B., Ulrich, R., \& Bausenhart, K. M. (2006). Attention delays perceived stimulus offset. Vision Research, 46(18), 2926-2933. https://doi.org/10.1016/j.visres.2006.02.022

Saito, C., Janssen, S. M., \& Tayama, T. J. 基. (2015). The effects of performance feedback on interval timing: Learning and generalization. 34(1), 27-34.

Schindel, R., Rowlands, J., \& Arnold, D. H. (2011). The oddball effect: Perceived duration and predictive coding. Journal of Vision, 11(2), 17-17.

Steinborn, M. B., Rolke, B., Bratzke, D., \& Ulrich, R. (2008). Sequential effects within a short foreperiod context: evidence for the conditioning account of temporal preparation. Acta Psychol (Amst), 129(2), 297-307. https://doi.org/10.1016/j.actpsy.2008.08.005

Thee, K. W., Nisar, H., \& Soh, C. S. J. I. A. (2018). Graph theoretical analysis of functional brain networks in healthy subjects: visual oddball paradigm. IEEE Access, 6, 64708-64727.

Thomaschke, R., Wagener, A., Kiesel, A., \& Hoffmann, J. (2011). The scope and precision of specific temporal expectancy: evidence from a variable foreperiod paradigm. Attention, Perception, \& Psychophysics, 73(3), 953-964. https://doi.org/10.3758/s13414010-0079-1

Tse, P. U. (2010). Attention underlies subjective temporal expansion. Attention and time, 137-150.

Tse, P. U., Intriligator, J., Rivest, J., \& Cavanagh, P. (2004). Attention and the subjective expansion of time. Attention, Perception, \& Psychophysics, 66(7), 1171-1189.

Ulrich, R., Nitschke, J., \& Rammsayer, T. (2006). Perceived duration of expected and unexpected stimuli. Psychological Research, 70(2), $77-87$. 
Vallesi, A., \& Shallice, T. (2007). Developmental dissociations of preparation over time: deconstructing the variable foreperiod phenomena. J Exp Psychol Hum Percept Perform, 33(6), 1377-1388. https:// doi.org/10.1037/0096-1523.33.6.1377

Vallesi, A., Shallice, T., \& Walsh, V. (2007). Role of the Prefrontal Cortex in the Foreperiod Effect: TMS Evidence for Dual Mechanisms in Temporal Preparation. Cereb Cortex, 17(2), 466474. https://doi.org/10.1093/cercor/bhj163

Vallesi, A., McIntosh, A. R., \& Stuss, D. T. (2009). Temporal preparation in aging: A functional MRI study. Neuropsychologia, 47(13), 28762881.

Vallesi, A., Lozano, V. N., \& Correa, Á. (2013). Dissociating temporal preparation processes as a function of the inter-trial interval duration. Cognition, 127(1), 22-30. https://doi.org/10.1016/j.cognition.2012. 11.011

Valls-Solé, J., Kofler, M., Kumru, H., Castellote, J. M., \& Sanegre, M. T. (2005). Startle-induced reaction time shortening is not modified by prepulse inhibition. Exp Brain Res, 165(4), 541-548. https://doi.org/ $10.1007 / \mathrm{s} 00221-005-2332-8$

Valls-Solé, J., Solé, A., Valldeoriola, F., Muñoz, E., Gonzalez, L. E., \& Tolosa, E. S. (1995). Reaction time and acoustic startle in normal human subjects. Neurosci Lett, 195(2), 97-100. https://doi.org/10. 1016/0304-3940(94)11790-P

Walsh, M. M., Gunzelmann, G., \& Anderson, J. R. (2017). Relationship of P3b single-trial latencies and response times in one, two, and three-stimulus oddball tasks. Biological psychology, 123, 47-61. https://doi.org/10.1016/j.biopsycho.2016.11.011

Wearden, J. (1991). Do humans possess an internal clock with scalar timing properties? Learning and Motivation, 22(1), 59-83.

Wearden, J., Edwards, H., Fakhri, M., \& Percival, A. (1998). Why "sounds are judged longer than lights": Application of a model of the internal clock in humans. The Quarterly Journal of Experimental Psychology: Section B, 51(2), 97-120.

Wearden, J. H., \& Farrar, R. (2007). Effects of feedback and calibration on the verbal estimation of the duration of tones. Acta Psychologica, 126(1), 1-17. https://doi.org/10.1016/j.actpsy.2006.10.003

Wearden, J., \& Lejeune, H. (2008). Scalar properties in human timing: Conformity and violations. The Quarterly Journal of Experimental Psychology, 61(4), 569-587.
Wehrman, J. (2020a). The simultaneous oddball: Oddball presentation does not affect simultaneity judgments. Attention, Perception, \& Psychophysics https://doi.org/10.3758/s13414-019-01866-6

Wehrman, J. (2020b). Temporal productions in a variable environment: timing starts from stimulus identification rather than onset. Psychological Research https://doi.org/10.1007/s00426-02001430-0

Wehrman, J., \& Sowman, P. (2019). Associative learning of response inhibition affects perceived duration in a subsequent temporal bisection task. Acta Psychologica, 201, 102952. https://doi.org/10.1016/j. actpsy.2019.102952

Wehrman, J. J., Wearden, J., \& Sowman, P. (2018a). The expected oddball: effects of implicit and explicit positional expectation on duration perception. Psychological Research, 1-15.

Wehrman, J. J., Wearden, J. H., \& Sowman, P. (2018b). Short-term effects on temporal judgement: Sequential drivers of interval bisection and reproduction. Acta Psychologica, 185, 87-95. https://doi. org/10.1016/j.actpsy.2018.01.009

Wehrman, J. J., Kaplan, D. M., \& Sowman, P. F. (2020a). Local context effects in the magnitude-duration illusion: Size but not numerical value sequentially alters perceived duration. Acta Psychologica, 204, 103016. https://doi.org/10.1016/j.actpsy.2020.103016

Wehrman, J. J., Wearden, J., \& Sowman, P. (2020b). Decisional carryover effects in interval timing: Evidence of a generalized response bias. Attention, Perception \& Psychophysics, 1-18.

Wiener, M., Thompson, J. C., \& Coslett, H. B. (2014). Continuous Carryover of Temporal Context Dissociates Response Bias from Perceptual Influence for Duration. PLoS One, 9(6), e100803. https://doi.org/10.1371/journal.pone.0100803

Xuan, B., Chen, X.-C., He, S., \& Zhang, D.-R. (2009). Numerical magnitude modulates temporal comparison: An ERP study. Brain Res, $1269,135-142$.

Publisher's note Springer Nature remains neutral with regard to jurisdictional claims in published maps and institutional affiliations. 